\title{
Comportamiento de 50 familias de medios hermanos de Solanum quitoense Lam bajo selección recurrente
}

\section{Performance of $\mathbf{5 0}$ families of half sibs in Solanum quitoense Lam under recurrent selection}

\author{
Tulio C. Lagos ${ }^{1 *}$, Julián Apraez², Liz K. Lagos³, David E. Duarte4. \\ Recibido para publicación: Junio 3 de 2015 - Aceptado para publicación: Noviembre 2 de 2015
}

\begin{abstract}
RESUMEN
El presente estudio se orientó hacia la implementación de un programa de selección recurrente de medios hermanos, como base para el programa de mejoramiento genético de lulo de castilla en la zona Andina del departamento de Nariño. Se evaluaron 50 familias de medios hermanos en dos localidades (La Florida y Yacuanquer), con el fin de seleccionar aquellas que contribuyan a mejorar la producción, calidad y productividad del cultivo de lulo en el departamento de Nariño. Los ensayos se sembraron bajo el diseño de bloques completos al azar, con dos repeticiones. Se evaluaron las variables rendimiento, peso de fruto, peso de semilla por fruto, contenido de jugo, solidos solubles totales, acidez titulable e índice de madurez. Este primer ciclo de selección recurrente de familias de medios hermanos de lulo de Castilla fue efectivo, dado que se obtuvo un incremento en el rendimiento con 10,67 t ha-1, en el peso de fruto con 33,34 g y en contenido de jugo con $18 \mathrm{ml}$ para las familias LSCmmh22, LSCmmh06, LSCmmh36, LSCmmh32, LSCmmh44, LSCmmh08, LSCmmh42, LSCmmh45, LSCmmh34 y LSCmmh29, las cuales obtuvieron los mayores índices de selección.
\end{abstract}

Palabras clave: Fitomejoramiento, lulo de Castilla, interacción genotipo por ambiente, rendimiento, calidad.

\begin{abstract}
The present study was oriented towards the implementation of recurrent selection by half sibs as a basis for the breeding program of lulo de Castilla in the Andean zone of Nariño. 50 families of half sib were evaluated in two locations (Florida and Yacuanquer), in order to select those that improved production, quality and crop productivity of lulo in the department of Nariño. Trials were planted under randomized complete blocks design with two replications. Yield, fruit weight, seed weight per fruit, juice content, total soluble solids, titratable acidity and maturity index were evaluated. This first cycle of half sibs recurrent selection of lulo de Castilla was effective, since an increase was obtained in yield with $10,67 \mathrm{tha}^{-1}$, on the weight of fruit with $33,34 \mathrm{~g}$ and juice content with 18 $\mathrm{ml}$ for families LSCmmh22, LSCmmh06, LSCmmh36, LSCmmh32, LSCmmh44, LSCmmh08, LSCmmh42, LSCmmh45, LSCmmh34 and LSCmmh29, which obtained the highest rates of selection.
\end{abstract}

Key words: Plant breeding, lulo de Castilla, genotype by environment interaction, yield, quality.

\footnotetext{
1* Ingeniero Agrónomo Ph.D, Profesor Titular, Universidad de Nariño, Facultad de Ciencias Agrícolas, Pasto, Colombia; teléfono: 3146150292-7313315; correo electrónico: tclagos@yahoo.com.

2 Ingeniero Agrónomo, Docente hora cátedra, Universidad de Nariño, Pasto, Nariño.

${ }^{3}$ Ingeniera Agrónoma M.Sc, Docente hora cátedra, Universidad de Nariño, Pasto, Colombia.

${ }^{4}$ Ingeniero Agrónomo, Co-Investigador, Universidad de Nariño, Pasto, Colombia
} 


\section{INTRODUCCIÓN}

El cultivo de lulo de Castilla Solanum quitoense Lam es de gran importancia económica para la fruticultura andina de Colombia. Actualmente se siembran 7327 ha con un rendimiento de 9,4 t ha-1 (Agronet 2015), según el Plan Frutícola Nacional se requieren sembrar 10.000 ha hasta el 2025 (Tafur et al. 2006). Sin embargo, el cultivo se caracteriza por presentar un sin número de problemas en campo, siendo las plagas y las enfermedades, los limitantes más frecuentes en los sistemas productivos del país. Algunas plagas como el pasador del fruto, Neoleucinodes elegantalis ocasionan perdidas hasta del $90 \%$ en el cultivo de lulo (Díaz y Brochero 2012).

En el caso de las enfermedades, la mayoría de los cultivares han mostrado ser susceptibles a las enfermedades radiculares causadas por Fusarium spp. y Meloidogyne spp, Ios cuales causan la muerte de la planta en el primer caso y en el segundo caso debilitan la planta, reducen la vida útil del cultivo ocasionando pérdidas hasta del $70 \%$ y a su vez disminuyen su capacidad productiva (Tamayo 2001; Tamayo y Navarro 2003).

Por otro lado, existe una escasa oferta de cultivares mejorados que presenten tolerancia o resistencia a los patógenos antes mencionados, solo se cuenta con el híbrido interespecifico La Selva (Franco et al. 2002), que está orientado a la industria de los jugos, con nula adopción por los cultivadores de lulo del sur del País, debido a las exigencias del mercado como fruto de tamaño grande y de pulpa verde. Además, la falta de programas de mejoramiento genético en el cultivo de lulo, no ha permitido obtener soluciones duraderas, y la baja productividad de la especie en Nariño $\left(4,9 \mathrm{t} \mathrm{ha}^{-1}\right)$, con respecto a otros departamentos productores como el Huila o Antioquia (7,5 y $12,5 \mathrm{t} \mathrm{ha}^{-1}$ respectivamente), limita los ingresos de los agricultores, ocasionando la desaparición de grandes áreas de cultivo, tal como ha sucedido en muchas regiones del departamento. Por lo tanto, es necesario incrementar la productividad y sostenibilidad del cultivo de lulo, partiendo con una línea estratégica de generación y evaluación de germoplasma promisorio (variedades, clones, líneas e híbridos) para el sur occidente de Colombia. Igualmente, una comprensión científica de los factores bióticos y abióticos que determinan la productividad de la planta, permitirá mejorar la oferta de genotipos mejor adaptados que garanticen mejorar la competitividad de los productores con frutas de calidad para el consumidor directo o para la industria.

Ante la escases de genotipos mejorados, el programa de mejoramiento genético de lulo (S. quitoense) para la zona productora del departamento de Nariño del GPFA (Grupo de Investigación en Producción de Frutales Andinos), plantea la selección recurrente de medios hermanos $(\mathrm{MH})$ como una estrategia para la producción permanente de poblaciones mejoradas, con resistencia a los principales enfermedades radiculares como Fusarium oxysporum y con altos rendimientos aprovechando el potencial productivo del lulo de Castilla. De esta manera, se podrán incrementar los ingresos de los productores de esta solanácea.

En los métodos de selección recurrente, se llevan a cabo ciclos alternantes de selección y cruzamiento. La selección con el fin de elevar la frecuencia de genes favorables en la población referencia y el cruzamiento entre sí, de las plantas seleccionadas, para mantener la variabilidad genética que permita obtener las mejores combinaciones hibridas (Ramírez 2006).

En 1964 Lonnquist desarrollo la metodología de mazorca por surco modificado, el cual es un sistema de selección entre y dentro de familias de medios hermanos $(\mathrm{MH})$ sin cruzamiento de 
prueba. La selección entre familias de $\mathrm{MH}$ se basa en el comportamiento promedio de cada una de las familias, las cuales se comparan con el promedio de la población. La selección dentro de familias de $\mathrm{MH}$ se basa en el comportamiento individual de cada planta, la cual se compara con el promedio de la familia a que pertenece. La evaluación y selección de las mejores familias se hace a través de ensayos de campo en una o varias localidades. Este método involucra dos unidades de selección diferente, en las cuales se controla el progenitor femenino. Cada ciclo de selección requiere de dos épocas de siembra (Vega 1988).

Paterniani (1967) modificó el método de Lonnquist, realizando en la segunda fase, la recombinación de las mejores familias de $\mathrm{MH}$, es decir, aquellas que fueron seleccionadas. A diferencia del esquema anterior, las familias de $\mathrm{MH}$ seleccionadas se siembran en surcos que actúan como progenitores femeninos. Estos, se alternan con los surcos del progenitor masculino, que provienen de la mezcla balanceada de las familias de $\mathrm{MH}$ seleccionadas.

En consideración con lo anterior, el objetivo de este trabajo fue evaluar 50 familias de medios hermanos (FMH) de lulo de Castilla, Solanum quitoense Lam., con base en su comportamiento agronómico bajo dos condiciones ambientales de clima frio moderado del departamento de Nariño, sur de Colombia.

\section{MATERIALES Y MÉTODOS}

Localización. El presente trabajo se realizó en dos municipios del departamento de Nariño, que presentan condiciones ambientales contrastantes en cuanto a altitud y tipo de suelos. La vereda Chapacual, municipio de Yacuanquer, está ubicada a 2450 msnm. Tiene un $10,9 \%$ de materia orgánica, un $\mathrm{pH}$ del suelo de 5,5 (ácido), bajo contenido de fosforo $\left(12,1 \mathrm{mg} \mathrm{kg}^{-1}\right)$ y bajo contenido de potasio $(0,64$ cmolcarga $/ \mathrm{kg})$. La vereda Santa Ana, corregimiento de Matituy, se localiza a 1980 msnm, su suelo tiene un contenido medio de materia orgánica $(6,24 \%)$, es ácido $(\mathrm{pH}=5,3)$, bajos contenidos de fosforo $\left(6,8 \mathrm{mg} \mathrm{kg}^{-1}\right)$ y potasio $(0,54 \mathrm{cmolcarga} / \mathrm{kg})$.

Familia de medios hermanos (FMH). Las FMH provienen de una población experimental tipo Castilla LSCm, formada a partir de una introducción realizada en el año 2008 desde la Unidad de Recursos Filogenéticos de CORPOICA La Selva, Antioquia. Esta población se originó, plantas sobrevivientes de un cultivo de lulo de Castilla afectado por Fusarium oxysporum en el Centro de Investigaciones La Selva de CORPOICA. De la semilla resultante de estas plantas, se hicieron tres siembras sucesivas en Matituy, La Florida, donde han mostrado tolerancia a dicha enfermedad, prevalente en esta región. En el semestre $A$ de 2010, de una población de 300 plantas se seleccionaron 50 por su buen comportamiento, relacionado con la tolerancia a Fusarium oxysporum, adecuado tamaño de fruto y alto rendimiento. De cada planta, se tomó un fruto; esté corresponde a una familia de medios hermanos, que experimentalmente toman el código de LSCmmh\# (Lulo de la Selva tipo Castilla, primer ciclo sembrado en Matituy, familia de medio hermano serial que va desde LSCmmh1 hasta LSCmmh50).

Diseño experimental. En cada una de las dos localidades, mencionadas anteriormente, se sembraron dos ensayos bajo un diseño de Bloques Completos al Azar con 53 tratamientos y dos repeticiones. Los tratamientos correspondieron a las 50 familias LSCmmh\#, y un cultivar regional tipo castilla utilizado como testigo. La parcela experimental estuvo formada por un surco de cuatro plantas. Las plantas se sembraron a 2,50 m entre ellas, con separación entre surcos de 2,50 m para una superficie de $25 \mathrm{~m}^{2}$. Para la parcela útil, se consideraron tres plantas centrales, que ocuparon un área de $18,75 \mathrm{~m}^{2}$. 


\section{Variables evaluadas}

Componentes de rendimiento. Se evaluaron el peso de semilla por fruto (PSF) y el peso de fruto en cuatro frutos por cada cosecha (PF).

Rendimiento (RTO). Con base en la producción de la parcela útil (tres plantas centrales, cada 15 días y durante siete meses, de cada ensayo se hizo la recolección de frutos maduros, para finalmente expresar el rendimiento en $t h^{-1}$ año.

Contenido de jugo (C)). Con base en 10 frutos de la parcela útil, se extrajo el jugo (zumo) de fruta, exprimiendo directamente, por extracción mecánica a través de licuadora mecánica y se expresó en $\mathrm{ml} /$ fruto. Igualmente, se midió el $\mathrm{pH}$ del jugo obtenido.

Sólidos solubles totales (SST). Se utilizó el método refractométrico. Se expresa en grados Brix $\left({ }^{\circ} \mathrm{Bx}\right)$. La lectura se corrigió utilizando el porcentaje de ácido cítrico (A.C), mediante la ecuación: SST=0,194×A.C+S.S.T.

Acidez titulable o porcentaje de ácido cítrico (A.C). Se determinó por el método de titulación potenciométrica. Se expresa como porcentaje de ácido cítrico y se calcula mediante la siguiente ecuación:

$\% A . C=((V 1 \times N) / V 2)) \times K \times 100$.

Dónde:

$\mathrm{V} 1=$ Volumen de $\mathrm{NaOH}$ consumido $(\mathrm{ml})$

$\mathrm{V} 2=$ Volumen de la muestra $(3 \mathrm{ml})$

$\mathrm{K}=$ peso equivalente del ácido cítrico $(0,064 \mathrm{~g}$ meq $^{-1}$ )

$\mathrm{N}=$ normalidad del $\mathrm{NaOH}\left(0,1 \mathrm{meq}^{-1}\right)$.

Indice de madurez (IM). Es la relación entre los SST y la acidez titulable.

Análisis de la Información. Las variables evaluadas se sometieron al Análisis de Varianza
(ANDEVA) combinado bajo un modelo mixto, siendo las localidades de efecto aleatorio y los genotipos de efecto fijo. Para probar los genotipos, el test de F se calculó con base en la interacción genotipo x ambiente. Cuando esta interacción no fue significativa, solo se tuvo en cuenta el ANDEVA combinado y la prueba de hipótesis de los efectos fijos se probaron contra un nuevo error experimental o error agrupado $(E A)$, que es igual a:

$\mathrm{EA}=(\mathrm{S} . \mathrm{C}$ genotipo $\mathrm{x}$ ambiente + S.C error $) /$ (G.L genotipo $x$ ambiente + G.L error)

En las variables donde la interacción genotipo $x$ ambiente fue significativa, se analizó el comportamiento de las FMH en cada una de las dos localidades. Cuando se encontraron diferencias significativas entre FMH para cualquier variable evaluada, la comparación de medias se hizo con base en lo planteado por Antuna et al. (2003) y De la Cruz et al. (2010). Aquellos individuos con promedios que presentaron diferencias estadísticas a los demás, son los que superaron a la media general más una vez el error estándar $(\mu+\sigma)$ o dos veces su correspondiente error estándar $(\mu+2 \sigma)$.

Para seleccionar el $20 \%$ de las FMH se aplicó un índice de selección (IS). Para establecer el IS, en primer lugar, se estandarizaron (E) los valores de las variables que hicieron parte del IS, mediante la ecuación: $E=\left[\left(X_{i j}-m\right) / s\right]$, donde: $X i j=$ observación individual, $\mathrm{m}=$ promedio general de cada FMH por variable y $\mathrm{s}=$ desviación estándar. Una vez se estandarizaron los datos, se estableció el IS, así:

$\mathrm{IS}=(\mathrm{RTO} \quad \mathrm{x} \quad 0,5)+(\mathrm{PF} \quad \mathrm{x} \quad 0,2)+(\mathrm{SST} \quad \mathrm{x}$ $0,1)+(\mathrm{CJ} * 0,05)+(\mathrm{IM} \times 0,05)-\left(\mathrm{PSF}^{*} 0,1\right)$

Dónde:

$\mathrm{RTO}=$ rendimiento económico ( $\mathrm{kg} /$ planta $/ \mathrm{mes})$ $\mathrm{PF}=$ peso de frutos $(\mathrm{g})$

SST $=$ sólidos solubles totales $\left({ }^{\circ} \mathrm{Bx}\right)$

$\mathrm{CJ}=$ Contenido de jugo por fruto $(\mathrm{ml})$ 
$\mathrm{IM}=$ índice de madurez

$\mathrm{PSF}=$ peso de semilla seca por fruto $(\mathrm{g})$

\section{RESULTADOS Y DISCUSIÓN}

Análisis de Varianza (ANDEVA) Combinado. En la Tabla 1 se presentan los cuadrados medios del ANDEVA combinado, para las variables contenido de jugo por fruto (CJ), acidez titulable (AC), solidos solubles totales (SST), índice de madurez (IM), peso de semilla por fruto (PSF), peso de fruto (PF) y rendimiento (RTO), evaluadas en $50 \mathrm{FMH}$ de $S$. quitoense a través de dos localidades de la región andina del departamento de Nariño.

En las variables AC, SST, IM, PSF Y RTO, no se presentaron diferencias significativas entre las FMH. La AC a través de las localidades, osciló entre 0,9 a $1,71 \%$ con una media de $1,25 \pm$ 0,06 . En la tabla 2 , se presenta una correlación negativa significativa de -0,55 entre las variables AC e IM, dado que el IM varía dependiendo del contenido de ácidos orgánicos del fruto. Según la norma ICONTEC NTC 5093 (ICONTEC 2002), el contenido máximo de ácido cítrico determinado mediante el método de titulación potenciométrica es de 3,23\%. Por lo tanto, las familias seleccionadas para la evaluación presentan valores dentro de los parámetros de acidez. Sin embargo, es necesario tener en cuenta que el lulo de Castilla es climatérico para procesos de comercialización, lo que reduce su tiempo de almacenamiento hasta su consumo.

Los SST oscilan entre $7,04{ }^{\circ} \mathrm{Bx}$ (LSCmmh25) y $10,56^{\circ} \mathrm{Bx}$ (LCSmmh8), con una media de 8,71 $\pm 0,73$. El $54 \%$ de los genotipos evaluados se encuentra en el rango de 8,5 a $10,1{ }^{\circ} \mathrm{Bx}$ correspondiente a los estados de maduración 4 y 5 de la norma NTC 5093 (ICONTEC 2002). Sin embargo, Muñoz (2010) afirma que los atributos deseables en el fruto, no están ligados estrictamente a un solo carácter y se debe tener en cuenta variables como color de pulpa y peso de fruto para lograr la aceptación en otros mercados. Esta variable presenta una correlación significativa con el IM de 0,79 (Tabla 2). En otra especie relacionada como Cyphomandra betacea (tomate de árbol), Lagos et al. (2013) encontraron una correlación

Tabla 1. Cuadrados medios del ANDEVA para contenido de jugo por fruto (CJ), acidez titulable (AC), solidos solubles totales (SST), índice de madurez (IM), peso de semilla por fruto (PSF), peso de fruto (PF) y rendimiento (RTO), evaluadas en 50 FMH de S. quitoense a través de dos localidades de la región Andina del departamento de Nariño.

\begin{tabular}{|c|c|c|c|c|c|c|c|c|}
\hline FV & GL & $\begin{array}{c}\mathrm{CJ} \\
(\mathrm{ml})\end{array}$ & $\begin{array}{l}\mathrm{AC} \\
(\%) \\
\end{array}$ & $\begin{array}{c}\text { SST } \\
\left({ }^{\circ} \mathbf{B x}\right)\end{array}$ & IM & $\begin{array}{l}\text { PSF } \\
(\mathrm{g}) \\
\end{array}$ & $\begin{array}{l}\text { PF } \\
(\mathrm{g})\end{array}$ & 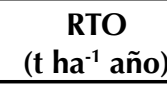 \\
\hline Localidad (Loc) & 1 & $4282,03^{*}$ & 0,01 & 6,66 & 1,68 & 7,14 & 874,75 & 57,43 \\
\hline Bloque /Loc & 2 & $1413,27^{*}$ & 0,001 & 0,79 & 0,57 & 2,51 & 771,75 & 7,89 \\
\hline Genotipo & 50 & $312,66^{*}$ & 0,013 & 1,70 & 1,58 & 1,65 & $845,8^{*}$ & 45,33 \\
\hline Genotipo X Loc & 47 & 223,29 & 0,022 & 1,55 & 1,70 & 1,90 & 550,52 & $33,90^{*}$ \\
\hline Error & 71 & $207,29 E A$ & $0,019^{\mathrm{EA}}$ & $1,46^{\mathrm{EA}}$ & $1,55^{\mathrm{EA}}$ & $1,90^{\mathrm{EA}}$ & $540,30^{\text {ЕA }}$ & 2,60 \\
\hline Media & & 46,58 & 1,25 & 8,80 & 7,11 & 2,77 & 81,69 & 9,57 \\
\hline $\mathbf{R}^{2}$ & & 0,72 & 0,61 & 0,61 & 0,60 & 0,59 & 0,65 & 0,96 \\
\hline CV & & 30,11 & 10,20 & 13,44 & 16,93 & 49,41 & 28,27 & 16,84 \\
\hline
\end{tabular}

$\mathrm{R}^{2}=$ coeficiente de determinación para el modelo del ANDEVA, EA = error agrupado, $\mathrm{CV}=$ coeficiente de determinación $(\%), *$ diferencias significativas o significancia de la interacción Genotipo x Loc. 
Tabla 2. Correlaciones significativas de Pearson entre las variables evaluadas en $50 \mathrm{FMH}$ a través de dos localidades de la región natural Andina del departamento de Nariño.

\begin{tabular}{lccccccc}
\hline & CJ & AC & IM & PSF & PF & SST & RTO \\
\hline CJ & 1 & & & 0,34 & $0,58^{*}$ & $0,79^{*}$ & 0,31 \\
AC & & 1 & $-0,55^{*}$ & & & & \\
IM & & & 1 & & 0,15 & 0,17 & 0,17 \\
PSF & & & 1 & $0,39^{*}$ & 1 & \\
PF & & & & 1 & & $0,50^{*}$ \\
SST & & & & & & & 0,17 \\
RTO & & & & & & 1 \\
\hline
\end{tabular}

${ }^{*}=$ significancia con $\alpha<0.05$

fenotípica y ambiental significativa entre estas dos variables $(0,61 *$ y $0,69 *$, respectivamente), sin embargo, no se encontró una correlación genética significativa, afirmando que el ambiente es el que influye en la expresión de este carácter.

Para la variable IM, los valores se encuentran entre 5,29 y 8,95 con una media de $7,07 \pm$ 0,71 . Esta variable se encuentra ligada a la relación entre SST y AC. Reina et al. (1998) determinaron que los SST aumentan con el proceso de maduración del fruto y la acidez. Estas tres variables son útiles al momento de determinar el máximo climatérico en lulo, y deben estar asociadas a otras variables de calidad para determinar con exactitud el grado de madurez en que se encuentra el fruto.

Para PSF y PF, los valores se encuentran entre 0,70 y 4,00 g y entre 16,36 y 119,69 $\mathrm{g}$, respectivamente; además, presentan una correlación significativa $\left(\mathrm{PF}_{\mathrm{vs}} \mathrm{PSF}=0,39\right)$. Para PF se obtuvieron diferencias significativas entre genotipos y las familias evaluadas presentan una media de 81,46 $\pm 16,36$ g. Según Muñoz (2010) el tamaño y el peso del fruto son las características más relevantes para todos los actores de la cadena de lulo. Sin embargo, productores de lulo afirman que solo frutos con pesos por encima de $100 \mathrm{~g}$ son aptos para consumo en fresco. Acorde con lo anterior, cuatro de las familias evaluadas cumplen con esta condición (LSCmmh28, LSCmmh32, LSCmmh37 y LSCmmh44) y el PSF promedio es 2,73 g, lo cual es considerado bajo y permitiría utilizarlos en procesos de industrialización.

La variable RTO presenta diferencias significativas, con valores que oscilan entre 3,31 y 17,94 tha ${ }^{-1}$ año, una media de 9,57 \pm 3,55 $\mathrm{t} \mathrm{ha}^{-1}$ año y una correlación significativa con el PF de 0,50 (Tabla 2). Según Agronet (2015) el rendimiento promedio nacional para el año 2013 fue de 9,4 t ha-1, lo que indica que algunas de las familias evaluadas alcanzan este promedio, sin embargo, familias como LSCmmh6 muestran un promedio de 17,94 t ha $^{-1}$ y LSCmmh22 un promedio de 16,88 $\mathrm{t} \mathrm{ha}^{-}$ 1. Estas dos familias se deben considerar para realizar procesos de mejoramiento genético, evaluando su potencial productivo y otras características de importancia agronómica como resistencia a enfermedades.

Para CJ se encontraron diferencias significativas (Tabla 1) entre genotipos y presenta una media de 43,278 $\pm 8,16$. Se observó una correlación significativa con el PF $(0,58)$, lo que sugiere que una selección por PF, incide de manera directa en el aumento o reducción de contenido de jugo. La familia LSCmmh22 obtuvo el mayor promedio de $68,13 \mathrm{ml}$ y el menor promedio la familia LSCmmh31 con 19,66 ml. Esto significa que para obtener $1 \mathrm{~L}$ de jugo en el caso del genotipo LSCmmh22, se necesitan 15 frutos y para LSCmmh31 se necesitan 51 frutos.

En la tabla 3 se observan los promedios de las variables contenido de jugo (CJ), acidez titulable (AC), solidos solubles totales (SST), índice de madurez (IM), peso de semillas por fruto (PSF) y peso del fruto (PF) de $50 \mathrm{FMH}$ 
Tabla 3. Promedio de contenido de jugo (CJ) en ml, acidez titulable (AC), solidos solubles totales (SST) en 'brix, índice de madurez (IM), peso de semillas por fruto (PSF) en g y peso del fruto (PF) en g de 50 familias de medios hermanos de lulo de Castilla evaluados a través de dos localidades de la región natural Andina del departamento de Nariño 2014.

\begin{tabular}{|c|c|c|c|c|c|c|c|c|c|c|c|c|c|}
\hline FMH & CJ & $\mathrm{AC}$ & SST & IM & PSF & PF & FMH & CJ & $\mathrm{AC}$ & SST & IM & PSF & PF \\
\hline LSCmmh1 & 31,38 & 1,28 & 8,74 & 6,91 & 2,62 & 75,45 & LSCmmh29 & $353,50 *$ & 1,21 & 9,88 & 8,29 & 1,79 & 82,68 \\
\hline LSCmmh2 & 47,63 & 1,18 & 8,06 & 6,88 & 2,58 & 87,23 & LSCmmh30 & 30,41 & 1,17 & 7,88 & 6,69 & 2,56 & 69,05 \\
\hline LSCmmh3 & 32,50 & 1,11 & 8,47 & 7,57 & 3,78 & 73,83 & LSCmmh31 & 19,66 & 1,35 & 8,54 & 6,37 & 2,78 & 56,22 \\
\hline LSCmmh4 & 41,13 & 1,25 & 7,88 & 6,29 & 3,57 & 61,48 & LSCmmh32 & $53,07 *$ & 1,22 & 9,83 & 8,08 & 2,86 & $119,69 * *$ \\
\hline LSCmmh5 & 35,00 & 1,32 & 9,51 & 7,22 & 2,47 & 49,12 & LSCmmh33 & NE & NE & NE & NE & NE & NE \\
\hline LSCmmh6 & $52,82 *$ & 1,25 & 9,78 & 7,84 & 3,91 & $99,15^{*}$ & LSCmmh34 & 49,41 & 1,30 & 8,69 & 6,66 & 1,75 & 83,84 \\
\hline LSCmmh7 & 43,88 & 1,22 & 9,34 & 7,75 & 1,87 & 81,91 & LSCmmh35 & 47,16 & 1,26 & 8,11 & 6,45 & 1,19 & 81,02 \\
\hline LSCmmh8 & 36,75 & 1,28 & 10,56 & 8,36 & 2,75 & 76,36 & LSCmmh36 & $58,50^{*}$ & 1,30 & 9,13 & 7,12 & 3,19 & 96,37 \\
\hline LSCmmh9 & NE & NE & NE & NE & NE & NE & LSCmmh37 & $52,68^{*}$ & 1,27 & 8,97 & 6,98 & 3,65 & $100,45^{*}$ \\
\hline LSCmmh10 & 33,97 & 1,31 & 8,86 & 7,11 & 3,57 & 54,95 & LSCmmh38 & 46,25 & 1,31 & 8,32 & 6,47 & 1,96 & 82,14 \\
\hline LSCmmh11 & NE & NE & NE & NE & NE & NE & LSCmmh39 & 42,93 & 1,15 & 9,24 & 8,11 & 3,68 & 77,85 \\
\hline LSCmmh13 & 46,59 & 1,23 & 8,83 & 7,26 & 3,29 & 90,43 & LSCmmh40 & 50,50 & 1,28 & 8,94 & 7,08 & 3,05 & 82,66 \\
\hline LSCmmh12 & 42,68 & 1,34 & 9,14 & 6,93 & 2,03 & 57,85 & LSCmmh41 & 46,32 & 1,19 & 8,10 & 6,84 & 4,00 & 90,64 \\
\hline LSCmmh14 & 39,00 & 1,37 & 7,95 & 5,83 & 2,66 & 53,07 & LSCmmh42 & 49,93 & 1,18 & 8,60 & 7,27 & 2,52 & 93,25 \\
\hline LSCmmh15 & 50,18 & 1,19 & 8,62 & 7,22 & 2,61 & 87,85 & LSCmmh43 & 44,68 & 1,27 & 9,38 & 7,53 & 3,35 & 63,75 \\
\hline LSCmmh16 & $57,66^{*}$ & 1,14 & 8,55 & 7,74 & 1,87 & 77,16 & LSCmmh44 & $59,75^{* *}$ & 1,22 & 8,11 & 6,73 & 3,28 & $109,72^{*}$ \\
\hline LSCmmh17 & $53,68 *$ & 1,25 & 8,04 & 6,43 & 2,68 & $98,40^{*}$ & LSCmmh45 & 49,50 & 1,29 & 8,98 & 7,00 & 3,17 & 99,36* \\
\hline LSCmmh18 & 47,50 & 1,29 & 8,78 & 6,91 & 1,96 & 75,90 & LSCmmh46 & 38,16 & 1,37 & 8,51 & 6,25 & 3,40 & 81,22 \\
\hline LSCmmh19 & 41,41 & 1,31 & 7,47 & 5,79 & 1,97 & 89,24 & LSCmmh47 & 51,00 & 1,29 & 9,95 & 7,92 & 2,36 & 82,72 \\
\hline LSCmmh20 & 29,16 & 1,23 & 8,85 & 7,26 & 2,53 & 75,92 & LSCmmh48 & 43,84 & 1,21 & 9,13 & 7,56 & 1,80 & 60,4 \\
\hline LSCmmh21 & $64,58^{* *}$ & 1,26 & 7,78 & 6,14 & 2,35 & $103,17^{*}$ & LSCmmh49 & 41,50 & 1,27 & 8,67 & 6,84 & 3,40 & 78,13 \\
\hline LSCmmh22 & $68,13^{* *}$ & 1,15 & 10,13 & 8,95 & 3,81 & $107,81^{*}$ & LSCmmh50 & 40,50 & 1,21 & 9,07 & 7,53 & 2,24 & 73,07 \\
\hline LSCmmh23 & 41,50 & 1,17 & 7,89 & 6,81 & 2,83 & 76,49 & Testigo & 43,00 & 1,19 & 8,76 & 7,42 & 1,85 & 71,55 \\
\hline LSCmmh24 & $51,88^{*}$ & 1,24 & 9,02 & 7,26 & 3,02 & 90,34 & & & & & & & \\
\hline LSCmmh25 & 42,91 & 1,33 & 7,04 & 5,29 & 3,46 & 85,49 & & 43,27 & & & & & 81,46 \\
\hline LSCmmh26 & $54,13^{*}$ & 1,24 & 8,25 & 6,65 & 2,52 & 95,83 & $\sigma \mathbf{g}$ & 8,16 & 1,25 & 8,71 & 7,07 & 2,73 & 16,36 \\
\hline LSCmmh27 & 27,75 & 1,15 & 7,73 & 6,73 & 1,80 & 49,11 & $\mu g+\sigma g$ & 51,43 & 0,06 & 0,73 & 0,71 & $\mathbf{0 , 7}$ & 97,82 \\
\hline LSCmmh28 & $57,43^{*}$ & 1,19 & 8,10 & 6,88 & 2,91 & $100,67^{*}$ & $\begin{array}{l}\mu \mathrm{g}+\sigma \mathrm{g} \\
\mu \mathrm{g}+2 \sigma \mathrm{g}\end{array}$ & 59,59 & & & & & 114,17 \\
\hline
\end{tabular}

$\mu \mathrm{g}=$ media general de las 50 FMH; $\sigma \mathrm{g}$ = desviación estándar de las 50 FMH.

de lulo de Castilla evaluadas a través de dos localidades de la región natural Andina del departamento de Nariño.

En las variables CJ y PF existieron diferencias significativas entre los promedios (Tabla 3). Para CJ, las familias LSCmmh21 $(64,58 \mathrm{ml})$, LSCmmh22 $(68,13 \mathrm{ml})$, LSCmmh44 $(59,75 \mathrm{ml})$, LSCmmh16 (57,66ml), LSCmmh28 $(57,43 \mathrm{ml})$, LSCmmh26 (54,13ml), LSCmmh17 $(53,68 \mathrm{ml})$, LSCmmh29 (53,50ml), LSCmmh32 $(53,07 \mathrm{ml})$, LSCmmh6 (52,82 ml), LSCmmh37 $(52,68 \mathrm{ml})$ y LSCmmh24 $(51,88 \mathrm{ml})$ con un promedio de 41,33 \pm 8,19 ml obtuvieron los valores más altos, superando estadísticamente al 74,51\% de las familias incluida el testigo tipo Castilla que obtuvo un promedio de $43 \mathrm{ml}$.
La familia LSCmmh31 fue la de más bajo valor de CJ con 19,66 ml. Esta variable es de importancia agronómica, dado que el lulo se utiliza para la industria de jugos a nivel nacional, y pueden seleccionarse las familias con los mayores promedios para su agroindustrialización.

Para PF, las familias LSCmmh32 (119,69 g), LSCmmh44 (109,72 g), LSCmmh22 (107,81 g), LSCmmh21 (103,17 g), LSCmmh28 (100,67 g), LSCmmh37 (100,45 g), LSCmmh45 (99,36 g) LSCmmh6 (99,15 g) y LSCmmh17 (98,40 g) alcanzaron los valores más altos con una media de $77,76 \pm 13,49 \mathrm{~g}$, superando estadísticamente al $82,35 \%$ de las familias incluido el testigo $(71,55 \mathrm{~g})$ y a LSCmmh27 que consiguió el promedio más bajo con un peso de 49,11 g. 
En la tabla 4 se observan los rendimientos (t ha $^{-1}$ año) de las $50 \mathrm{FMH}$ de lulo de Castilla, evaluados en Chapacual (Yacuanquer), Matituy (La Florida) y a través de estas localidades. En Chapacual, las familias con los rendimientos $(t$ ha $^{-1}$ año) más altos fueron LSCmmh22 $\left(24,80^{* *}\right)$, LSCmmh8 (18,68*) LSCmmh6 (17,65*), LSCmmh33 (16,96*), LSCmmh19 (15,36*), LSCmmh41 $\left(14,13^{*}\right), \operatorname{LSCmmh16}\left(13,81^{*}\right)$ y el promedio más bajo con un rendimiento de 1,3 $\mathrm{t} \mathrm{ha}^{-1}$ año pertenece LSCmmh50.

En la localidad de Matituy, las familias con mayores rendimientos (t/ha/año) corresponden a LSCmmh36 (21,33**), LSCmmh21 $\left(19,78^{*}\right)$, LSCmmh6 (18,23*), LSCmmh42 (18,03*), LSCmmh45 (17,54*), LSCmmh44 (16,97*), LSCmmh24 $\left(16,51^{*}\right), \quad$ LSCmmh28 $\left(15,94^{*}\right)$,
LSCmmh34 $\left(15,11^{*}\right)$. Estas familias superan el promedio nacional de 9,4 $\mathrm{t} \mathrm{ha}^{-1}$ (Agronet 2015) y al promedio del testigo de 4,99 $\mathrm{t} \mathrm{ha}^{-1}$ año. El rendimiento más bajo lo exhibió la familia LSCmmh16 con 2,02 tha-1 año.

En la tabla 5 se observan los promedios de las variables que componen el índice de selección (IS) de la fracción (20\%) seleccionada de 50 FMH de lulo de Castilla. Para RTO, se obtuvo una media general de 9,57 t ha-1 año, sin embargo, al aplicar el índice de selección, la media de la fracción seleccionada $\left(\bar{y}_{s}\right)$ fue de $14,70 \mathrm{t}$ ha $^{-1}$ año con un diferencial de selección (DS) de 5,13 tha-1 año, lo cual indica una ganancia en el rendimiento. La familia LSCmmh32 presenta el rendimiento más bajo con 11,31 t ha-1 año y el valor máximo con 17,94 t ha $^{-1}$

Tabla 4. Rendimientos promedio ( $\mathrm{ha} \mathrm{h}^{-1}$ año) de $50 \mathrm{FMH}$ de lulo de Castilla, evaluados en Chapacual (Yacuanquer), Matituy (La Florida) y a través de estas localidades 2014.

\begin{tabular}{|c|c|c|c|c|c|c|c|}
\hline FMH & Chapacual & Matituy & Promedio & FMH & Chapacual & Matituy & Promedio \\
\hline LSCmmh1 & 5,29 & 8,70 & 6,99 & LSCmmh29 & 10,51 & 13,41 & 11,96 \\
\hline LSCmmh2 & 6,06 & 8,46 & 7,26 & LSCmmh30 & 7,78 & 13,69 & 10,74 \\
\hline LSCmmh3 & 2,69 & 7,60 & 5,14 & LSCmmh31 & 6,82 & 12,83 & 9,83 \\
\hline LSCmmh4 & 7,04 & 4,88 & 5,96 & LSCmmh32 & 13,12 & 9,51 & 11,31 \\
\hline LSCmmh5 & 2,55 & 11,18 & 6,86 & LSCmmh33 & $16,96^{*}$ & NE & NE \\
\hline LSCmmh6 & $17,65^{*}$ & $18,23^{*}$ & $17,94^{* *}$ & LSCmmh34 & 13,14 & $15,11^{*}$ & 14,13 \\
\hline LSCmmh7 & 5,00 & 11,36 & 8,18 & LSCmmh35 & 11,13 & 11,4 & 11,26 \\
\hline LSCmmh8 & $18,68^{*}$ & 10,41 & $14,54^{*}$ & LSCmmh36 & 11,67 & $21,33^{* *}$ & $16,50^{*}$ \\
\hline LSCmmh9 & 1,48 & NE & NE & LSCmmh37 & 4,53 & 9,34 & 6,93 \\
\hline LSCmmh10 & 6,27 & 4,13 & 5,2 & LSCmmh38 & 9,01 & 11,80 & 10,40 \\
\hline LSCmmh11 & 6,06 & NE & NE & LSCmmh39 & 12,59 & 2,97 & 7,78 \\
\hline LSCmmh13 & 3,44 & 11,14 & 7,29 & LSCmmh40 & 8,01 & 14,46 & 11,23 \\
\hline LSCmmh12 & 7,42 & 5,50 & 6,46 & LSCmmh41 & $14,13^{*}$ & 6,86 & 10,49 \\
\hline LSCmmh14 & 2,11 & 5,26 & 3,68 & LSCmmh42 & 11,43 & $18,03^{*}$ & $14,73^{*}$ \\
\hline LSCmmh15 & 9,95 & 8,18 & 9,06 & LSCmmh43 & 10,30 & 7,06 & 8,68 \\
\hline LSCmmh16 & $13,81^{*}$ & 2,02 & 7,92 & LSCmmh44 & 12,41 & $16,97^{*}$ & $14,69 *$ \\
\hline LSCmmh17 & 7,93 & 7,10 & 7,52 & LSCmmh45 & 11,09 & $17,54 *$ & $14,31 *$ \\
\hline LSCmmh18 & 11,43 & 8,45 & 9,94 & LSCmmh46 & 9,57 & 13,78 & 11,67 \\
\hline LSCmmh19 & $15,36^{*}$ & 8,14 & 11,75 & LSCmmh47 & 6,62 & 2,86 & 4,74 \\
\hline LSCmmh20 & 13,08 & 7,31 & 10,2 & LSCmmh48 & 3,02 & 9,72 & 6,37 \\
\hline LSCmmh21 & 3,74 & $19,78^{*}$ & 11,76 & LSCmmh49 & 11,57 & 7,32 & 9,44 \\
\hline LSCmmh22 & $24,80^{* *}$ & 8,96 & $16,88^{* *}$ & LSCmmh50 & 1,30 & 10,62 & 5,96 \\
\hline LSCmmh23 & 7,03 & 12,97 & 10,00 & Testigo & 4,39 & 5,59 & 4,99 \\
\hline LSCmmh24 & 3,69 & $16,51^{*}$ & 10,10 & & & & \\
\hline LSCmmh25 & 9,36 & 8,69 & 9,03 & $\mu \mathrm{g}$ & 8,91 & 10,2 & 10,2 \\
\hline LSCmmh26 & 8,54 & 3,70 & 6,12 & $\sigma \mathbf{g}$ & 4,90 & 4,82 & 4,82 \\
\hline LSCmmh27 & 3,95 & 2,67 & 3,31 & $\boldsymbol{\mu g}+\sigma \mathrm{g}$ & 13,80 & 15,02 & 15,02 \\
\hline LSCmmh28 & 8,78 & $15,94 *$ & 12,36 & $\mu g+2 \sigma g$ & 18,70 & 19,85 & 19,85 \\
\hline
\end{tabular}

$\mu \mathrm{g}=$ media general de las $50 \mathrm{FMH} ; \sigma \mathrm{g}=$ desviación estándar de las 50 FMH; NE = No Evaluado.. 
Tabla 5. Promedio de las variables que componen el índice de selección (IS) de la fracción (20\%) seleccionada de $50 \mathrm{FMH}$ de lulo de Castillo evaluadas en dos ambientes de la región andina del departamento de Nariño 2014.

\begin{tabular}{|c|c|c|c|c|c|c|c|c|}
\hline No. & FMH & RTO & PF & SST & CJ & IM & PSF & IS \\
\hline 1 & LSCmmh22 & 16,88 & 107,81 & 10,13 & 68,13 & 8,95 & 3,81 & 1,64 \\
\hline & LSCmmh06 & 17,94 & 99,15 & 9,78 & 52,82 & 7,84 & 3,91 & 1,46 \\
\hline & LSCmmh36 & 16,50 & 96,37 & 9,13 & 58,50 & 7,12 & 3,19 & 1,22 \\
\hline 41 & LSCmmh32 & 11,31 & 119,69 & 9,83 & 53,07 & 8,08 & 2,86 & 0,96 \\
\hline & LSCmmh44 & 14,69 & 109,72 & 8,11 & 59,75 & 6,73 & 3,28 & 0,95 \\
\hline & LSCmmh08 & 14,54 & 76,36 & 10,56 & 36,75 & 8,36 & 2,75 & 0,93 \\
\hline & LSCmmh42 & 14,73 & 93,25 & 8,60 & 49,93 & 7,27 & 2,52 & 0,92 \\
\hline & LSCmmh45 & 14,31 & 99,36 & 8,98 & 49,50 & 7,00 & 3,17 & 0,88 \\
\hline & LSCmmh34 & 14,13 & 83,84 & 8,68 & 49,41 & 6,66 & 1,75 & 0,80 \\
\hline $10 \mathrm{I}$ & LSCmmh29 & 12,36 & 100,67 & 9,88 & 57,43 & 8,29 & 2,91 & 0,77 \\
\hline $\bar{y}_{s}$ & & 14,70 & 96,82 & 9,37 & 53,53 & 7,63 & 3,01 & \\
\hline $\mathbf{S}$ & & 2,05 & 13,43 & 0,78 & 8,30 & 0,78 & 0,63 & \\
\hline$\mu g$ & & 9,57 & 81,46 & & & 7,07 & 2,73 & \\
\hline$\sigma \mathrm{g}$ & & 3,55 & 16,36 & 0,86 & 10,40 & 0,71 & 0,70 & \\
\hline DS & & 5,13 & 15,36 & 0,66 & 8,11 & 0,56 & 0,17 & \\
\hline
\end{tabular}

$\overline{\mathrm{y}}_{\mathrm{S}}=$ media de la fracción seleccionada; $\mathrm{S}=$ desviación estándar de la fracción seleccionada; $\mu \mathrm{g}=$ media general de las $50 \mathrm{FMH}$; $\sigma \mathrm{g}=$ desviación estándar de las $50 \mathrm{FMH} ; \mathrm{DS}=$ diferencial de selección $\left(\bar{y}_{s}-\mu g\right)$.

año, corresponde a la familia LSCmmh06. Estos resultados muestran posibilidades de avanzar en las ganancias genéticas en el rendimiento de esta población. Al respecto, Vallejo et al. (2012) afirman que el mejoramiento poblacional consiste en estudiar el material básico, su composición y estructura genética, con miras a incrementar el progreso a corto o largo plazo. Esto permite obtener nuevas poblaciones que incrementen la medida de rendimiento después de cada ciclo de selección, y que dicho incremento se debe a que los individuos seleccionados poseen genes superiores, que al recombinarse al azar, producen genotipos de mayor producción, por tanto se espera que la población sea más productiva en promedio que la anterior.

Para PF y PSF, la familia LSCmmh32 fue la de mayor peso promedio de fruto $(119,69 \mathrm{~g})$ con un peso de semilla de 2,86 g. El valor más bajo de PF correspondió a la familia LSCmmh08 (76,36 g) con un PSF de 2,75 g. Con base en el DS para ambas variables, se logró una ganancia en el PF de 15,36 g debido a la fracción seleccionada y una ganancia de 0,17 g para la variable PSF considerada baja y de utilidad para de procesos agroindustriales. Además, teniendo en cuenta el PF, las familias seleccionadas se encuentran entre los calibres 35, 30 y 26 (diámetro 51 a $>61 \mathrm{~mm}$ ) según la norma NTC 5093. El calibre indica el número de frutos que ocupan un área de $40 \times 30 \mathrm{~cm}$, por cada rango de diámetro y se pueden clasificar entre categorías extra a categoría I (ICONTEC 2002).

Para SST, la media general fue de $8,45^{\circ} \mathrm{Bx}$, una $\bar{y}_{\mathrm{S}}$ de $9,37^{\circ} \mathrm{Bx}$ y un DS de 0,66. La familia LSCmmh08 consiguió el mayor promedio con un valor de $10,56^{\circ} \mathrm{Bx}$ y la familia LSCmmh44 el menor promedio con un valor de 8,11 ${ }^{\circ} \mathrm{Bx}$. Esta variable presenta una correlación positiva significativa con IM de 0,79* (Tabla 2), demostrando que al realizar una selección por el contenido de SST $\left({ }^{\circ} \mathrm{Bx}\right)$, incide de manera directa en el aumento o reducción del IM. Por otro lado, las familias LSCmmh22 y LSCmmh44 adquirieron los promedios más altos de CJ $(68,13$ y $59,75 \mathrm{ml}$, respectivamente). Las 10 familias seleccionadas presentaron un DS de $8,11 \mathrm{ml}$, con una $\bar{y}_{\mathrm{s}}$ de $53,53 \mathrm{ml}$ en comparación con la media general de 45,03 ml. Esto indica que al aplicar el índice de selección, se ganó en el contenido de jugo y al tener una correlación significativa (Tabla 2) con el PF $(0,58)$ y RTO $(0,31)$, se puede afirmar que una selección por $\mathrm{CJ}$, influye en el aumento o reducción del PF y/o RTO.

\section{CONCLUSIONES}

El primer ciclo de selección recurrente aplicado en las 50 familias de medios hermanos de lulo de Castilla fue efectivo, dado que seleccionando las familias LSCmmh22, LSCmmh06, LSCmmh36, LSCmmh32, LSCmmh44, LSCmmh08, LSCmmh42, LSCmmh45, LSCmmh34 y LSCmmh29, se logró un incremento de 10,67 
t ha ${ }^{-1}$ año con respecto al testigo y de $6,26 \mathrm{t}$ $\mathrm{ha}^{-1}$ año con respecto al promedio nacional. Para el peso de fruto y contenido de jugo, se obtuvo un incremento de 33,34 g y $18 \mathrm{ml}$, en su orden, respecto al testigo. El rendimiento esta correlacionado de manera positiva $y$ significativa con el peso del fruto.

Se recomienda para el municipio de Yacuanquer, vereda Chapacual, las familias que presentaron alto rendimiento y superaron al testigo, las cuales corresponden a LSCmmh22, LSCmmh8, LSCmmh6, LSCmmh33, LSCmmh19, LSCmmh41 y LSCmmh16. Para el municipio de La Florida, vereda Matituy las familias LSCmmh36, LSCmmh21, LSCmmh6, LSCmmh42， LSCmmh45， LSCmmh44, LSCmmh24, LSCmmh28 y LSCmmh34.

Con base en el índice de selección (IS) compuesto por el rendimiento, el peso de fruto, los sólidos solubles totales, el contenido de jugo, el índice de madurez y el peso de semilla por fruto, las mejores familias con un IS superior a 0,77 fueron LSCmmh22, LSCmmh06, LSCmmh36, LSCmmh32, LSCmmh44, LSCmmh08, LSCmmh42, LSCmmh45, LSCmmh34 y LSCmmh29, las cuales se recomiendan para la etapa de recombinación en el siguiente ciclo de selección recurrente.

\section{REFERENCIAS}

Agronet. 2015. Red de información y comunicación del sector agropecuario Colombia. http://www.agronet.gov.co/ www/htm3b/ReportesAjax/VerReporte. aspx. [09/11/2015].

Antuna, O., Rincón, F., Gutiérrez del Rio, E., Ruiz, A. y Bustamante, L. 2003. Componentes genéticos de caracteres agronómicos y de calidad fisiológica de semillas de líneas de maíz. Rev. Fitotec. Mex. 26(1): 11-17.
De la Cruz, L., Castañón, G., Brito, N., Gómez, A., Robledo, V. y Lozano, A. 2010. Heterosis y aptitud combinatoria de poblaciones de maíz tropical. Revista Internacional de Botánica Experimental. 79:11-17

Díaz, A., Brochero, H. 2012. Parasitoides asociados al perforador del fruto de las solanáceas Neoleucinodes elegantalis (Lepidoptera: Crambidae) en Colombia. Revista Colombiana de Entomología 38 (1): p. 50-57

Franco, G., Bernal, E., Giraldo, C., Tamayo, P., Castaño, P., Tamayo, V., Gallego, J., Botero, M., Rodríguez, J., Guevara, N., Morales, J., Londoño, M., Ríos, G., Rodríguez, J., Cardona, J., Zuleta, O., Castaño, Z. y Ramírez, M. 2002. El Cultivo del Lulo. Manizales, Asohofrucol, Corpoica. 103 p.

ICONTEC. INSTITUTO COLOMBIANO DE NORMAS TECNICAS. 2002. Norma técnica Colombiana NTC 5093: Frutas frescos. Lulo de Castilla. Especificaciones. Bogotá, ICONTEC. CENICAFE. 19p.

Lagos, L., Vallejo, F., Lagos, T., Duarte, D. 2013. Correlaciones genotípicas, fenotípicas y ambientales, y análisis de sendero en tomate de árbol (Cyphomandra betacea Cav. Sendt.). Acta Agronómica. 62(3): 215-222.

Lonnquist, J. 1964. A modification of the Earto-row procedure for the improvement of maize populations. Crop Science 4(2):227-228.

Muñoz, L. 2010. Evaluación agronómica de materiales de lulo solanum $\mathrm{sp}$, frutal de alto potencial para zonas tropicales. Universidad Nacional de Colombia, sede Palmira. Trabajo de grado para optar al título de Magister. $89 \mathrm{p}$. 
Paterniani, E. 1967. Selection among and within half-sib families in a Brazilian population of maize (Zea mays L.). Crop Science. 7(3): 212-216.

Ramírez, L. 2006. Mejora de plantas alógamas. Universidad Pública de Navarra. Pamplona - España. pp. 14-16.

Reina, C., Araujo, C. y Manrique, I. 1998. Manejo pos cosecha y evaluación de la calidad del lulo (Solanum quitoense) que se comercializa en la ciudad de Neiva. Universidad Surcolombiana. Huila, Neiva. 141 p.

Tafur, R., Toro, J., Perfetti, J., Ruiz, D. y Morales, J. 2006. Plan Frutícola de ColombiaPFN. Cali, Fondo Nacional de fomento hortofrutícola, Asohofrucol, SAG. 43p.
Tamayo, P. 2001. Principales enfermedades del tomate de árbol, la mora y el lulo en Colombia. Boletín Técnico 12. Corporación Colombiana de Investigación agropecuaria. Rionegro, Antioquia. 44p

Tamayo, P. y Navarro, R. 2003. Enfermedades del cultivo de lulo en Colombia: guía de diagnóstico y control. Boletín técnico 18. Convenio Corpoica Bayer CropScience. Rionegro, Antioquia. 48p.

Vallejo, F., Estrada, E. 2012. Mejoramiento Genético de Plantas. Universidad Nacional de Colombia Sede Palmira. Valle del Cauca, Palmira. P. 268.

Vega, U. 1988. Mejoramiento genético de plantas. Editorial América, C.A. Maracay Venezuela. 200 p. 